\title{
Pharmacological Characterization of Standard Analgesics on Oxaliplatin- Induced Acute Cold Hypersensitivity in Mice
}

\author{
Meng Zhao ${ }^{1}$, Saki Nakamura ${ }^{1}$, Takahito Miyake ${ }^{1}$, Kanako So $^{1}$, Hisashi Shirakawa ${ }^{1}$, Shogo Tokuyama ${ }^{2,3}$, \\ Minoru Narita ${ }^{3,4}$, Takayuki Nakagawa ${ }^{1,3,5, *}$, and Shuji Kaneko ${ }^{1}$ \\ ${ }^{1}$ Department of Molecular Pharmacology, Graduate School of Pharmaceutical Sciences, Kyoto University, \\ 46-29 Yoshida-Shimoadachi-cho, Sakyo-ku, Kyoto 606-8501, Japan \\ ${ }^{2}$ Department of Clinical Pharmacy, School of Pharmaceutical Sciences, Kobe Gakuin University, \\ 1-1-3 Minatojima, Chuo-ku, Kobe 650-8586, Japan \\ ${ }^{3}$ Research Promotion Committee, Japanese Society for Pharmaceutical Palliative Care and Sciences, Tokyo, Japan \\ ${ }^{4}$ Department of Pharmacology, Hoshi University School of Pharmacy and Pharmaceutical Sciences, \\ 2-4-41 Ebara, Shinagawa-ku, Tokyo 142-8501, Japan \\ ${ }^{5}$ Department of Clinical Pharmacology and Therapeutics, Kyoto University Hospital, \\ 54 Shogoin-Kawahara-cho, Sakyo-ku, Kyoto 606-8507, Japan
}

Received November 26, 2013; Accepted February 17, 2014

\begin{abstract}
Oxaliplatin, a platinum-based chemotherapeutic agent, causes an acute peripheral neuropathy triggered by cold in almost all patients during or within hours after its infusion. We recently reported that a single administration of oxaliplatin induced cold hypersensitivity $2 \mathrm{~h}$ after the administration in mice. In this study, we examined whether standard analgesics relieve the oxaliplatin-induced acute cold hypersensitivity. Gabapentin, tramadol, mexiletine, and calcium gluconate significantly inhibited and morphine and milnacipran decreased the acute cold hypersensitivity, while diclofenac and amitriptyline had no effects. These results suggest that gabapentin, tramadol, mexiletine, and calcium gluconate are effective against oxaliplatin-induced acute peripheral neuropathy.
\end{abstract}

Keywords: oxaliplatin, acute peripheral neuropathy, analgesic

Some chemotherapeutic agents including platinum compounds, taxanes, vinca alkaloids, and bortezomib, cause peripheral neuropathy. However, unlike other chemotherapeutic agents, oxaliplatin, a platinum-based chemotherapeutic agent, induces a peculiar acute peripheral neuropathy, such as paresthesia and dysesthesia, which are often triggered or enhanced by exposure to cold, whereas cumulative and chronic neuropathy induced by oxaliplatin includes pain sensation (1). The acute peripheral neuropathy is induced in almost all patients during or within hours after the infusion. However, an effective pharmacological strategy for its management remains controversial $(1,2)$.

Many studies in animal models focus on the oxaliplatininduced chronic and/or subacute painful peripheral

*Corresponding author. tnakaga@pharm.kyoto-u.ac.jp

Published online in J-STAGE on March 27, 2014

doi: 10.1254 /jphs.13249SC neuropathy that appear several days to several weeks after oxaliplatin administration (3 - 5), while oxaliplatininduced acute peripheral neuropathy is poorly characterized. Recently, we have reported a mouse model of rapid-onset cold hypersensitivity induced by oxaliplatin (6). When mice are given a single administration of oxaliplatin, cold but not mechanical hypersensitivity is induced within $2 \mathrm{~h}$ after the administration, while it is not induced by other chemotherapeutic agents such as cisplatin and paclitaxel. These findings suggest that rapid-onset cold hypersensitivity is representative of the acute peripheral neuropathy characteristic to oxaliplatin in mice. In the present study, we assessed the effects of standard analgesics, such as non-steroidal anti-inflammatory agent (NSAID), opioid analgesics, tricyclic antidepressant, serotonin and noradrenaline reuptake inhibitor (SNRI), calcium channel $\alpha_{2}-\delta$ ligand, local anesthetic, and calcium gluconate $(1,2)$, on the oxaliplatin-induced acute cold hypersensitivity in mice. 
This study was carried out in strict accordance with the recommendations in the Guiding Principles for the Care and Use of The Japanese Pharmacological Society. The protocol was approved by the Kyoto University Animal Research Committee. The male C57BL/6J mice aged between $6-8$ weeks (Japan SLC, Shizuoka) were housed under constant ambient temperature $\left(24^{\circ} \mathrm{C} \pm 1^{\circ} \mathrm{C}\right)$, with alternate light-dark cycles. Food and water were freely available.

Oxaliplatin (Wako Pure Chemical Industries, Osaka) was freshly dissolved in $5 \%$ glucose solution. Mice received a single intraperitoneal administration of oxaliplatin $(5 \mathrm{mg} / \mathrm{kg})$ or vehicle $2 \mathrm{~h}$ before behavioral tests (6). Morphine hydrochloride (Takeda Pharmaceutical Co., Osaka); amitriptyline hydrochloride (LKT Laboratories, MN, USA); milnacipran hydrochloride (Santa Cruz Biotechnology, Santa Cruz, CA, USA); tramadol hydrochloride (gift from Nippon Shinyaku, Kyoto); diclofenac sodium salt, gabapentin, and mexiletine hydrochloride (Sigma-Aldrich, St. Louis, MO, USA) were freshly dissolved in sterile saline. Morphine and tramadol were administrated subcutaneously; milnacipran and mexiletine were administrated intraperitoneally 30 min before; and diclofenac, gabapentin, and amitriptyline were administered intraperitoneally $1 \mathrm{~h}$ before the behavioral test at a volume of $10 \mathrm{ml} / \mathrm{kg}$. The doses of these analgesics were chosen based on previous reports $(3-5$, 7 -11). Calcium gluconate monohydrate (Wako) was freshly dissolved in 5\% glucose solution and was infused intravenously just before oxaliplatin administration (5).

Cold sensitivity was assessed with the hot/cold-plate analgesimeter (Ugo Basile, Milan, Italy) as previously described (6). Briefly, mice were individually placed on a cold plate maintained at $5^{\circ} \mathrm{C}$. Escape behaviors were graded with a score of $0=$ no response, $1=$ moderate effort to avoid cold, and 2 =vigorous effort to escape cold. The sum of the scores recorded within a 60 -s period was calculated.

The data are presented as means \pm S.E.M. Statistical significance was calculated by one-way analyses of variance (ANOVA), followed by the Tukey-Kramer post-hoc test. In all cases, differences of $P<0.05$ were considered statistically significant.

Consistent with our previous study (6), the score of cold escape behaviors was significantly increased $2 \mathrm{~h}$ after a single intraperitoneal administration of oxaliplatin, compared with the vehicle-treated group (Fig. 1: A - I), suggesting that oxaliplatin produces rapid-onset cold hypersensitivity.

An NSAID, diclofenac (25 and $50 \mathrm{mg} / \mathrm{kg}$ ) had no effect on the oxaliplatin-induced cold hypersensitivity (Fig. 1A). NSAIDs are representative broad-spectrum analgesics especially for nociceptive pain, while they are ineffective on neuropathic pain. Although NSAIDs are initially used for the management of chemotherapyinduced painful peripheral neuropathy (1), acute peripheral neuropathy is unlikely to include inflammatory components. The present results suggest that NSAIDs are ineffective on oxaliplatin-induced acute peripheral neuropathy.

The strong opioid analgesic morphine ( 5 and $10 \mathrm{mg} / \mathrm{kg}$ ) tended to inhibit the cold hypersensitivity (Fig. 1B). It is reported that morphine $(1-4 \mathrm{mg} / \mathrm{kg})$ dose-dependently inhibits the chronic and subacute cold allodynia induced by oxaliplatin in rats $(3,4)$. However, the present results suggest that morphine is less efficacious against oxaliplatin-induced acute peripheral neuropathy, which may include an aspect of A-fibers-mediated sensations other than pain (12).

A calcium channel $\alpha_{2}-\delta$ ligand, gabapentin (10 and $30 \mathrm{mg} / \mathrm{kg}$ ), dose-dependently decreased the cold hypersensitivity, and a significant difference was observed at $30 \mathrm{mg} / \mathrm{kg}$ (Fig. 1C). Gabapentin is often used as a firstchoice drug for the management of neuropathic pain, as well as pregabalin (13). Pregabalin reduces the severity of oxaliplatin-induced peripheral neuropathy in patients, although gabapentin has not well proven its efficacy (1). In animal models, gabapentin and pregabalin reduce oxaliplatin-induced chronic and/or subacute peripheral neuropathy $(3,4,7)$.

The tricyclic antidepressant amitriptyline (5 and 10 $\mathrm{mg} / \mathrm{kg}$ ) had no effect (Fig. 1D), while the SNRI milnacipran $(10$ and $30 \mathrm{mg} / \mathrm{kg})$ tended to inhibit the cold hypersensitivity (Fig. 1E). Tricyclic antidepressants and SNRIs are widely used as first-choice drugs for the management of neuropathic pain (13). Both antidepressants inhibit neuropathic pain by blocking noradrenaline and serotonin reuptake. However, their efficacy on the oxaliplatin-induced peripheral neuropathy is controversial (1). In animal models, repeated administration of amitriptyline reduces oxaliplatininduced chronic mechanical allodynia (8). It is considered that disinhibition and imbalance of the descending serotonergic and noradrenergic pain inhibitory pathways contribute to neuropathic pain. However, oxaliplatininduced acute peripheral neuropathy is unlikely to be mediated through such disinhibition and imbalance, which may result in no and weak efficacy of these antidepressants. On the other hand, the anti-allodynic effect of milnacipran is more potent than that of amitriptyline on cold allodynia in a neuropathic pain model rats (9), consistent with the present results.

Tramadol (10 and $20 \mathrm{mg} / \mathrm{kg}$ ) dose-dependently decreased the cold hypersensitivity, and a significant difference was observed at $20 \mathrm{mg} / \mathrm{kg}$ (Fig. 1F). It is clinically reported that tramadol/acetaminophen combination is 
A) Diclofenac

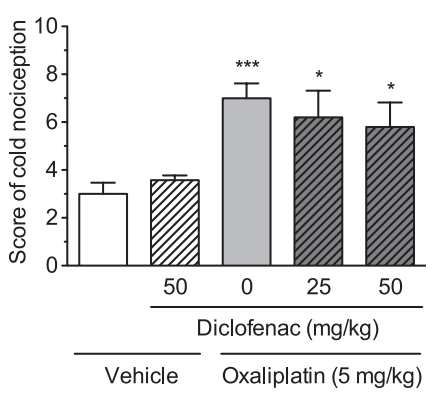

D) Amitriptyline

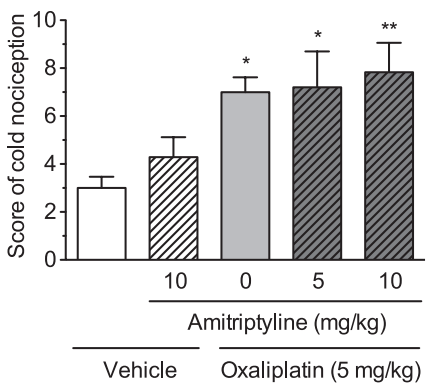

G) Morphine + Milnacipran

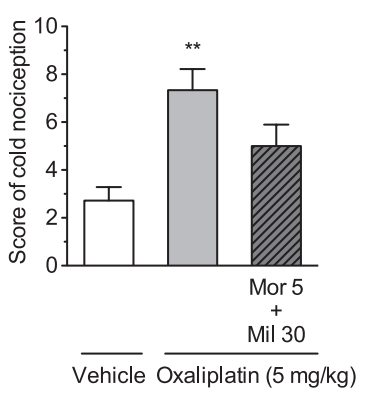

B) Morphine

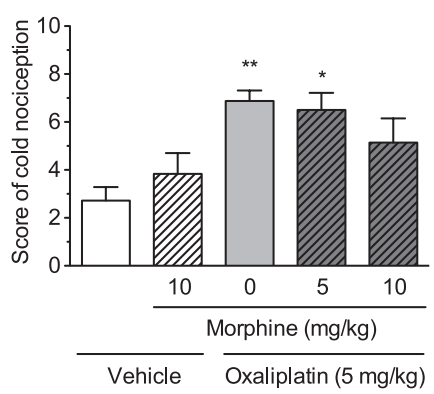

E) Milnacipran

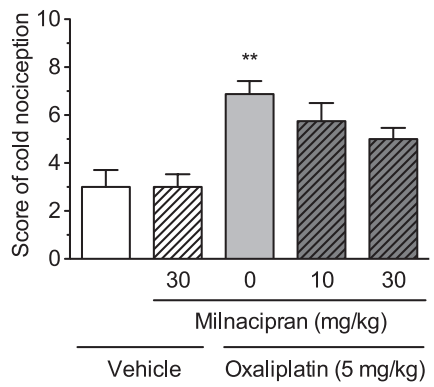

H) Mexiletine

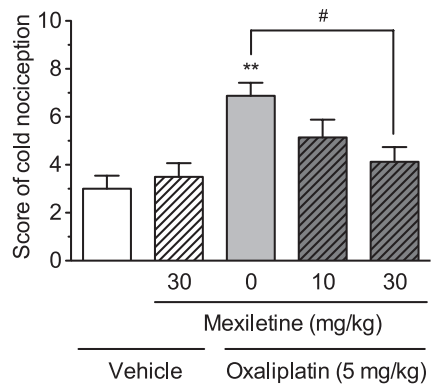

C) Gabapentine

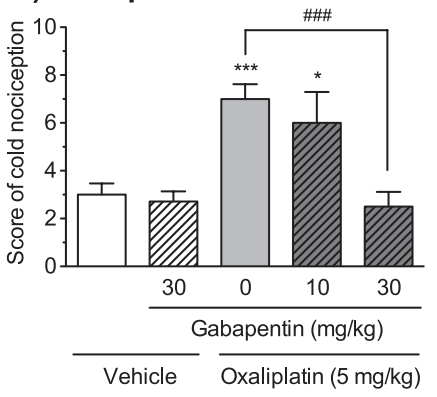

F) Tramadol

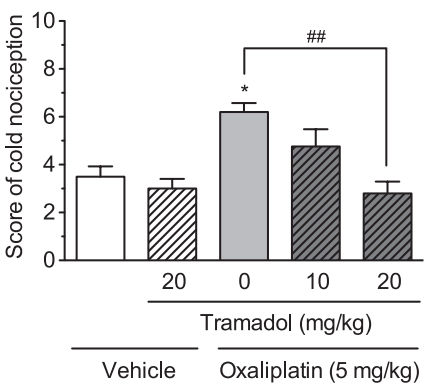

I) Calcium gluconate

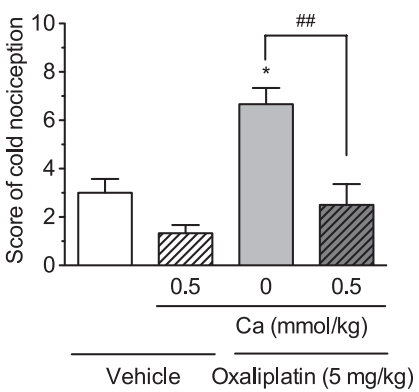

Fig. 1. Effects of analgesics on oxaliplatin-induced acute cold hypersensitivity. Mice were given intraperitoneal administration of oxaliplatin $(5 \mathrm{mg} / \mathrm{kg})$. Cold plate tests were performed $2 \mathrm{~h}$ after oxaliplatin administration. Morphine $(5 \mathrm{and} 10 \mathrm{mg} / \mathrm{kg}$, $\mathrm{n}=6-8)$, tramadol (10 and $20 \mathrm{mg} / \mathrm{kg}, \mathrm{n}=5-8)$, milnacipran (10 and $30 \mathrm{mg} / \mathrm{kg}, \mathrm{n}=4-8)$, mexiletine $(10 \mathrm{and} 30 \mathrm{mg} / \mathrm{kg}$, $\mathrm{n}=5-8)$, and combination of morphine $(5 \mathrm{mg} / \mathrm{kg})+$ milnacipran $(30 \mathrm{mg} / \mathrm{kg}, \mathrm{n}=3-6)$ were administered subcutaneously or intraperitoneally $30 \mathrm{~min}$ before the tests. Diclofenac ( 25 and $50 \mathrm{mg} / \mathrm{kg}, \mathrm{n}=5-9)$, gabapentin (10 and $30 \mathrm{mg} / \mathrm{kg}, \mathrm{n}=4-9)$, and amitriptyline ( 5 and $10 \mathrm{mg} / \mathrm{kg}, \mathrm{n}=5-9$ ) were administered intraperitoneally $60 \mathrm{~min}$ before the tests. Calcium gluconate $(0.5 \mathrm{mmol} / \mathrm{kg})$ was administered intravenously before oxaliplatin administration $(\mathrm{n}=3-4)$. Data are presented as the means \pm S.E.M. $* P<0.05, * * P<0.01, * * * P<0.001$ : compared to the group treated with vehicle alone instead of oxaliplatin. ${ }^{\#} P<0.05,{ }^{\#} P<0.01,{ }^{\# \# P} P<0.001$ (one-way ANOVA, followed by the Tukey-Kramer post-hoc test).

effective in the management of oxaliplatin-induced painful neuropathy (14). Tramadol exhibits an analgesic effect through both $\mu$-opioid receptor agonistic activity and antidepressant-like enhancement of descending serotoninergic and noradrenergic pain inhibitory pathway by blocking monoamine reuptake. Dual analgesic mechanisms of tramadol, acting both complementarily and synergistically, are suggested to contribute to its efficacy against neuropathic pain (10). However, the combination of morphine $(5 \mathrm{mg} / \mathrm{kg})$ and milnacipran $(30 \mathrm{mg} / \mathrm{kg}$ ) tended to inhibit the cold hypersensitivity, while the efficacy was weak, compared with tramadol (Fig. 1G). Therefore, dual action of opioids and antidepressants may only partly contribute to the efficacy against oxaliplatin-induced acute peripheral neuropathy. The efficacious mechanism of tramadol remains unclear, but it may be due to the inhibitory effect of tramadol on voltage-gated $\mathrm{Na}^{+}$channels (15).

The local anesthetic, mexiletine (10 and $30 \mathrm{mg} / \mathrm{kg})$ dose-dependently decreased the cold hypersensitivity, and a significant difference was observed at $30 \mathrm{mg} / \mathrm{kg}$ (Fig. $1 \mathrm{H})$. Mexiletine blocks voltage-gated $\mathrm{Na}^{+}$channels, 
and it is clinically used for painful diabetic neuropathy (13). An aspect of the oxaliplatin-induced peripheral neuropathy is mediated through voltage-gated $\mathrm{Na}^{+}$ channels in A-fibers (12). In animal models, the oxaliplatin-induced chronic cold allodynia is inhibited by mexiletine (11). Taken together, mexiletine is a potential treatment option for oxaliplatin-induced acute neuropathy.

Calcium gluconate $(0.5 \mathrm{mmol} / \mathrm{kg})$ significantly inhibited the cold hypersensitivity (Fig. 1I). Infusion of calcium and magnesium has been clinically used for prevention and management of oxaliplatin-induced peripheral neuropathy, although a recent meta-analysis does not support them (3). In animal models, calcium reduces oxaliplatin-induced chronic and/or subacute cold hyperalgesia but not mechanical allodynia $(4,5)$. It is hypothesized that chelation of calcium by an oxaliplatin metabolite, oxalate, induces functional impairment of voltage-gated $\mathrm{Na}^{+}$channels, resulting in hyperexcitability of sensory neurons. Therefore, calcium infusion could enhance the closing rate of $\mathrm{Na}^{+}$channels, which may decrease the neuronal hyperexcitability induced by oxaliplatin (12).

The present results show the efficacy of $\mathrm{Ca}^{2+}$ channel $\alpha_{2}-\delta$ ligands, tramadol, $\mathrm{Na}^{+}$-channel blockers, and calcium, rather than opioid analgesics, antidepressants, and NSAIDs, on the oxaliplatin-induced acute cold hypersensitivity. We previously reported that oxaliplatininduced acute cold hypersensitivity is caused by the enhanced responsiveness of transient receptor potential ankyrin 1 (TRPA1) expressed in primary sensory neurons (6). Furthermore, it includes an aspect of Afibers-mediated sensations (12). The present results suggest that the analgesics that have an ability to directly suppress the hyperexcitability of sensory neurons, including gabapentin, local anesthetic, and calcium, have higher efficacy than the centrally-acting analgesics activating the descending pain inhibitory pathway, including antidepressants, as well as opioids and gabapentin. The drug sensitivity of these analgesics further supports the possibility that oxaliplatin-induced cold hypersensitivity observed in mice may represent coldtriggered paresthesia and dysesthesia as clinical symptoms of oxaliplatin-induced acute peripheral neuropathy, rather than pain. The present study provides evidence for the treatment of cold-triggered acute peripheral neuropathy induced by oxaliplatin.

\section{Acknowledgments}

This work was supported by Grants-in-Aid for Scientific Research from the Ministry of Education, Culture, Sport, Science and Technology of Japan (No. 25670285) and the Salt Science Research Foundation, No. $13 \mathrm{C} 4$.

\section{Conflicts of Interest}

The authors indicated no potential conflicts of interest.

\section{References}

1 Ali BH. Amelioration of oxaliplatin neurotoxicity by drugs in humans and experimental animals: a mini review of recent literature. Basic Clin Pharmacol Toxicol. 2010;106:272-279.

2 Wu Z, Ouyang J, He Z, Zhang S. Infusion of calcium and magnesium for oxaliplatin-induced sensory neurotoxicity in colorectal cancer: a systematic review and meta-analysis. Eur J Cancer. 2012;48:1791-1798.

3 Ling B, Authier N, Balayssac D, Eschalier A, Coudore F. Behavioral and pharmacological description of oxaliplatininduced painful neuropathy in rat. Pain. 2007;128:225-234.

4 Ling B, Coudoré F, Decalonne L, Eschalier A, Authier N. Comparative antiallodynic activity of morphine, pregabalin and lidocaine in a rat model of neuropathic pain produced by one oxaliplatin injection. Neuropharmacology. 2008;55:724-728.

5 Sakurai M, Egashira N, Kawashiri T, Yano T, Ikesue H, Oishi R. Oxaliplatin-induced neuropathy in the rat: involvement of oxalate in cold hyperalgesia but not mechanical allodynia. Pain. 2009;147:165-174.

6 Zhao M, Isami K, Nakamura S, Shirakawa H, Nakagawa T, Kaneko S. Acute cold hypersensitivity characteristically induced by oxaliplatin is caused by the enhanced responsiveness of TRPA1 in mice. Mol Pain. 2012;8:55.

7 Gauchan P, Andoh T, Ikeda K, Fujita M, Sasaki A, Kato A, et al. Mechanical allodynia induced by paclitaxel, oxaliplatin and vincristine: different effectiveness of gabapentin and different expression of voltage-dependent calcium channel $\alpha_{2} \delta$-1 subunit. Biol Pharm Bull. 2009;32:732-734.

8 Sada H, Egashira N, Ushio S, Kawashiri T, Shirahama M, Oishi R. Repeated administration of amitriptyline reduces oxaliplatininduced mechanical allodynia in rats. J Pharmacol Sci. 2012; 118:547-551.

9 Berrocoso E, Mico JA, Vitton O, Ladure P, Newman-Tancredi A, Depoortère R, et al. Evaluation of milnacipran, in comparison with amitriptyline, on cold and mechanical allodynia in a rat model of neuropathic pain. Eur J Pharmacol. 2011;655:46-51.

10 Reeves RR, Burke RS. Tramadol: basic pharmacology and emerging concepts. Drugs Today (Barc). 2008;44:827-836.

11 Egashira N, Hirakawa S, Kawashiri T, Yano T, Ikesue H, Oishi R. Mexiletine reverses oxaliplatin-induced neuropathic pain in rats. J Pharmacol Sci. 2010;112:473-476.

12 Sittl R, Lampert A, Huth T, Schuy ET, Link AS, Fleckenstein J, et al. Anticancer drug oxaliplatin induces acute cooling-aggravated neuropathy via sodium channel subtype $\mathrm{Na}_{\mathrm{v}} 1.6$-resurgent and persistent current. Proc Natl Acad Sci U S A. 2012;109:6704-6709.

13 Dworkin RH, O’Connor AB, Backonja M, Farrar JT, Finnerup NB, Jensen TS, et al. Pharmacologic management of neuropathic pain: evidence-based recommendations. Pain. 2007;132:237-251.

14 Liu YC, Wang WS. Human mu-opioid receptor gene A118G polymorphism predicts the efficacy of tramadol/acetaminophen combination tablets (ultracet) in oxaliplatin-induced painful neuropathy. Cancer. 2012;118:1718-1725.

15 Haeseler G, Foadi N, Ahrens J, Dengler R, Hecker H, Leuwer M. Tramadol, fentanyl and sufentanil but not morphine block voltage-operated sodium channels. Pain. 2006;126:234-244. 